\title{
Inverse Trigonometric Functions Arcsec and Arccosec
}

\author{
Bing Xie \\ Qingdao University of Science \\ and Technology \\ China
}

\author{
Xiquan Liang \\ Qingdao University of Science \\ and Technology \\ China
}

\author{
Fuguo Ge \\ Qingdao University of Science \\ and Technology \\ China
}

\begin{abstract}
Summary. This article describes definitions of inverse trigonometric functions arcsec and arccosec, as well as their main properties.
\end{abstract}

MML identifier: $\underline{\text { SINCOS10, }}$, version: $\underline{7.8 .10 \quad 4.100 .1011}$

The papers [1], [2], [16], [3], [12], [17], [13], [5], [8], [11], [14], [4], [6], [7], [10], [15], and [9] provide the notation and terminology for this paper.

In this paper $x, r$ denote real numbers.

The following propositions are true:

(1) $\left[0, \frac{\pi}{2}[\subseteq \operatorname{dom}\right.$ (the function sec).

(2) $\left.] \frac{\pi}{2}, \pi\right] \subseteq \operatorname{dom}$ (the function sec).

(3) $\left[-\frac{\pi}{2}, 0[\subseteq \operatorname{dom}\right.$ (the function cosec).

(4) $\left.] 0, \frac{\pi}{2}\right] \subseteq \operatorname{dom}$ (the function cosec).

(5) The function sec is differentiable on $] 0, \frac{\pi}{2}[$ and for every $x$ such that $x \in] 0, \frac{\pi}{2}$ [ holds (the function sec) $)^{\prime}(x)=\frac{\sin x}{(\cos x)^{2}}$.

(6) The function sec is differentiable on $] \frac{\pi}{2}, \pi[$ and for every $x$ such that $x \in] \frac{\pi}{2}, \pi[\text { holds (the function sec) })^{\prime}(x)=\frac{\sin x}{(\cos x)^{2}}$.

(7)(i) The function cosec is differentiable on $]-\frac{\pi}{2}, 0[$, and 
(ii) for every $x$ such that $x \in]-\frac{\pi}{2}, 0[\text { holds (the function } \operatorname{cosec})^{\prime}(x)=$ $-\frac{\cos x}{(\sin x)^{2}}$

(8)(i) The function cosec is differentiable on $] 0, \frac{\pi}{2}[$, and

(ii) for every $x$ such that $x \in] 0, \frac{\pi}{2}\left[\right.$ holds (the function $\operatorname{cosec}^{\prime}(x)=$ $-\frac{\cos x}{(\sin x)^{2}}$.

(9) The function sec is continuous on $] 0, \frac{\pi}{2}[$.

(10) The function sec is continuous on $] \frac{\pi}{2}, \pi[$.

(11) The function cosec is continuous on $]-\frac{\pi}{2}, 0[$.

(12) The function cosec is continuous on $] 0, \frac{\pi}{2}[$.

(13) The function sec is increasing on $] 0, \frac{\pi}{2}[$.

(14) The function sec is increasing on $] \frac{\pi}{2}, \pi[$.

(15) The function cosec is decreasing on $]-\frac{\pi}{2}, 0[$.

(16) The function cosec is decreasing on $] 0, \frac{\pi}{2}[$.

(17) The function sec is increasing on $\left[0, \frac{\pi}{2}[\right.$.

(18) The function sec is increasing on $\left.] \frac{\pi}{2}, \pi\right]$.

(19) The function cosec is decreasing on $\left[-\frac{\pi}{2}, 0[\right.$.

(20) The function cosec is decreasing on $\left.] 0, \frac{\pi}{2}\right]$.

(21) (The function sec) $\uparrow\left[0, \frac{\pi}{2}[\right.$ is one-to-one.

(22) (The function sec) $\left.\uparrow \frac{\pi}{2}, \pi\right]$ is one-to-one.

(23) (The function cosec) $\uparrow\left[-\frac{\pi}{2}, 0[\right.$ is one-to-one.

(24) (The function cosec) \lceil] $\left.0, \frac{\pi}{2}\right]$ is one-to-one.

One can verify the following observations:

* (the function sec) $\uparrow\left[0, \frac{\pi}{2}\right.$ [ is one-to-one,

* (the function sec) $\left\lceil\frac{\pi}{2}, \pi\right]$ is one-to-one,

* (the function cosec) $\uparrow\left[-\frac{\pi}{2}, 0[\right.$ is one-to-one, and

* (the function cosec) \lceil] $\left.0, \frac{\pi}{2}\right]$ is one-to-one.

The partial function the 1st part of arcsec from $\mathbb{R}$ to $\mathbb{R}$ is defined as follows:

(Def. 1) The 1st part of $\operatorname{arcsec}=\left((\right.$ the function sec $) \uparrow\left[0, \frac{\pi}{2}[)^{-1}\right.$.

The partial function the 2 nd part of arcsec from $\mathbb{R}$ to $\mathbb{R}$ is defined as follows:

(Def. 2) The 2nd part of $\operatorname{arcsec}=\left((\right.$ the function sec $\left.\left.)\lceil] \frac{\pi}{2}, \pi\right]\right)^{-1}$.

The partial function the 1st part of arccosec from $\mathbb{R}$ to $\mathbb{R}$ is defined by:

(Def. 3) The 1st part of arccosec $=\left((\right.$ the function cosec $) \uparrow\left[-\frac{\pi}{2}, 0[)^{-1}\right.$.

The partial function the 2 nd part of arccosec from $\mathbb{R}$ to $\mathbb{R}$ is defined by:

(Def. 4) The 2 nd part of arccosec $=\left((\right.$ the function cosec $\left.\left.)\lceil] 0, \frac{\pi}{2}\right]\right)^{-1}$.

Let $r$ be a real number. The functor $\operatorname{arcsec}_{1} r$ is defined by:

(Def. 5) $\operatorname{arcsec}_{1} r=($ the 1st part of $\operatorname{arcsec})(r)$.

The functor $\operatorname{arcsec}_{2} r$ is defined as follows: 
(Def. 6) $\operatorname{arcsec}_{2} r=($ the 2 nd part of $\operatorname{arcsec})(r)$.

The functor $\operatorname{arccosec}_{1} r$ is defined as follows:

(Def. 7) $\operatorname{arccosec}_{1} r=$ (the 1st part of $\left.\operatorname{arccosec}\right)(r)$.

The functor $\operatorname{arccosec}_{2} r$ is defined by:

(Def. 8) $\operatorname{arccosec}_{2} r=($ the 2nd part of $\operatorname{arccosec})(r)$.

Let $r$ be a real number. Then $\operatorname{arcsec}_{1} r$ is a real number. Then $\operatorname{arcsec}_{2} r$ is a real number. Then $\operatorname{arccosec}_{1} r$ is a real number. Then $\operatorname{arccosec}_{2} r$ is a real number.

We now state four propositions:

(25) $\quad \operatorname{rng}($ the 1 st part of $\operatorname{arcsec})=\left[0, \frac{\pi}{2}[\right.$.

(26) $\quad \mathrm{rng}($ the 2 nd part of $\left.\operatorname{arcsec})=] \frac{\pi}{2}, \pi\right]$.

(27) $\mathrm{rng}($ the 1 st part of $\operatorname{arccosec})=\left[-\frac{\pi}{2}, 0[\right.$.

(28) $\quad \operatorname{rng}($ the 2 nd part of $\left.\operatorname{arccosec})=] 0, \frac{\pi}{2}\right]$.

One can check the following observations:

* the 1st part of arcsec is one-to-one,

* the 2nd part of arcsec is one-to-one,

* the 1st part of arccosec is one-to-one, and

* the 2nd part of arccosec is one-to-one.

Let $t_{1}$ be a real number. Then sec $t_{1}$ is a real number. Then $\operatorname{cosec}_{1}$ is a real number.

We now state a number of propositions:

(29) $\sin \left(\frac{\pi}{4}\right)=\frac{1}{\sqrt{2}}$ and $\cos \left(\frac{\pi}{4}\right)=\frac{1}{\sqrt{2}}$.

(30) $\sin \left(-\frac{\pi}{4}\right)=-\frac{1}{\sqrt{2}}$ and $\cos \left(-\frac{\pi}{4}\right)=\frac{1}{\sqrt{2}}$ and $\sin \left(\frac{3}{4} \cdot \pi\right)=\frac{1}{\sqrt{2}}$ and $\cos \left(\frac{3}{4} \cdot \pi\right)=$ $-\frac{1}{\sqrt{2}}$.

(31) $\sec 0=1$ and $\sec \left(\frac{\pi}{4}\right)=\sqrt{2}$ and $\sec \left(\frac{3}{4} \cdot \pi\right)=-\sqrt{2}$ and $\sec \pi=-1$.

(32) $\operatorname{cosec}\left(-\frac{\pi}{2}\right)=-1$ and $\operatorname{cosec}\left(-\frac{\pi}{4}\right)=-\sqrt{2}$ and $\operatorname{cosec}\left(\frac{\pi}{4}\right)=\sqrt{2}$ and $\operatorname{cosec}\left(\frac{\pi}{2}\right)=1$.

(33) For every set $x$ such that $x \in\left[0, \frac{\pi}{4}\right]$ holds $\sec x \in[1, \sqrt{2}]$.

(34) For every set $x$ such that $x \in\left[\frac{3}{4} \cdot \pi, \pi\right]$ holds $\sec x \in[-\sqrt{2},-1]$.

(35) For every set $x$ such that $x \in\left[-\frac{\pi}{2},-\frac{\pi}{4}\right]$ holds $\operatorname{cosec} x \in[-\sqrt{2},-1]$.

(36) For every set $x$ such that $x \in\left[\frac{\pi}{4}, \frac{\pi}{2}\right]$ holds $\operatorname{cosec} x \in[1, \sqrt{2}]$.

(37) The function sec is continuous on $\left[0, \frac{\pi}{2}[\right.$.

(38) The function sec is continuous on $\left.] \frac{\pi}{2}, \pi\right]$.

(39) The function cosec is continuous on $\left[-\frac{\pi}{2}, 0[\right.$.

(40) The function cosec is continuous on $\left.] 0, \frac{\pi}{2}\right]$.

(41) $\operatorname{rng}\left((\right.$ the function sec $)\left\lceil\left[0, \frac{\pi}{4}\right]\right)=[1, \sqrt{2}]$.

(42) $\operatorname{rng}\left((\right.$ the function sec $)\left\lceil\left[\frac{3}{4} \cdot \pi, \pi\right]\right)=[-\sqrt{2},-1]$. 
(43) $\operatorname{rng}\left((\right.$ the function cosec $)\left\lceil\left[-\frac{\pi}{2},-\frac{\pi}{4}\right]\right)=[-\sqrt{2},-1]$.

(44) $\operatorname{rng}\left((\right.$ the function cosec $)\left\lceil\left[\frac{\pi}{4}, \frac{\pi}{2}\right]\right)=[1, \sqrt{2}]$.

(45) $[1, \sqrt{2}] \subseteq$ dom (the 1st part of arcsec).

(46) $[-\sqrt{2},-1] \subseteq$ dom (the 2nd part of arcsec).

(47) $[-\sqrt{2},-1] \subseteq$ dom (the 1st part of arcosec).

(48) $[1, \sqrt{2}] \subseteq \operatorname{dom}($ the 2 nd part of arccosec).

One can check the following observations:

* (the function sec) $\uparrow\left[0, \frac{\pi}{4}\right]$ is one-to-one,

* (the function sec) $\uparrow\left[\frac{3}{4} \cdot \pi, \pi\right]$ is one-to-one,

* (the function cosec) $\uparrow\left[-\frac{\pi}{2},-\frac{\pi}{4}\right]$ is one-to-one, and

* (the function cosec) $\left\lceil\left[\frac{\pi}{4}, \frac{\pi}{2}\right]\right.$ is one-to-one.

One can prove the following propositions:

(49) (The 1st part of $\operatorname{arcsec}) \uparrow[1, \sqrt{2}]=\left((\right.$ the function sec $)\left\lceil\left[0, \frac{\pi}{4}\right]\right)^{-1}$.

(50) (The 2nd part of arcsec) $\uparrow[-\sqrt{2},-1]=\left((\right.$ the function sec $)\left\lceil\left[\frac{3}{4} \cdot \pi, \pi\right]\right)^{-1}$.

(51) (The 1st part of arcosec) $\uparrow[-\sqrt{2},-1]=($ (the function cosec) $)\left\lceil\left[-\frac{\pi}{2},-\frac{\pi}{4}\right]\right)^{-1}$.

(52) (The 2nd part of arccosec) $\uparrow[1, \sqrt{2}]=\left((\right.$ the function cosec $)\left\lceil\left[\frac{\pi}{4}, \frac{\pi}{2}\right]\right)^{-1}$.

(53) ((The function sec) $\uparrow\left[0, \frac{\pi}{4}\right]$ qua function $) \cdot(($ the 1 st part of arcsec $) \uparrow[1, \sqrt{2}])=$ $\operatorname{id}_{[1, \sqrt{2}]}$.

(54) ((The function sec) $\left\lceil\left[\frac{3}{4} \cdot \pi, \pi\right]\right.$ qua function) $\cdot($ the 2 nd part of $\operatorname{arcsec})\lceil[-\sqrt{2},-1])=\operatorname{id}_{[-\sqrt{2},-1]}$.

(55) ((The function cosec) $\uparrow\left[-\frac{\pi}{2},-\frac{\pi}{4}\right]$ qua function) $\cdot($ the 1 st part of $\operatorname{arccosec}) \uparrow[-\sqrt{2},-1])=\operatorname{id}_{[-\sqrt{2},-1]}$.

(56) ((The function cosec) $\uparrow\left[\frac{\pi}{4}, \frac{\pi}{2}\right]$ qua function) $\cdot(($ the 2 nd part of $\operatorname{arccosec})\lceil[1, \sqrt{2}])=\operatorname{id}_{[1, \sqrt{2}]}$.

(57) ((The function sec) $\left.\uparrow\left[0, \frac{\pi}{4}\right]\right) \cdot(($ the 1 st part of $\operatorname{arcsec}) \uparrow[1, \sqrt{2}])=\operatorname{id}_{[1, \sqrt{2}]}$.

(58) $\left((\right.$ The function sec $)\left\lceil\left[\frac{3}{4} \cdot \pi, \pi\right]\right) \cdot(($ the 2 nd part of arcsec $) \uparrow[-\sqrt{2},-1])=$ $\operatorname{id}_{[-\sqrt{2},-1]}$.

(59) $\left((\right.$ The function cosec $)\left\lceil\left[-\frac{\pi}{2},-\frac{\pi}{4}\right]\right) \cdot(($ the 1 st part of $\operatorname{arccosec}) \Upsilon[-\sqrt{2},-1])=$ $\operatorname{id}_{[-\sqrt{2},-1]}$.

(60) ((The function $\operatorname{cosec})\left\lceil\left[\frac{\pi}{4}, \frac{\pi}{2}\right]\right) \cdot(($ the 2 nd part of arccosec $) \uparrow[1, \sqrt{2}])=$ $\operatorname{id}_{[1, \sqrt{2}]}$.

(61) (The 1st part of arcsec qua function) $\cdot\left((\right.$ the function sec $) \uparrow\left[0, \frac{\pi}{2}[)=\right.$ $\operatorname{id}_{\left[0, \frac{\pi}{2}[\right.} \cdot$

(62) (The 2nd part of arcsec qua function) $\cdot\left((\right.$ the function sec $\left.\left.)\lceil] \frac{\pi}{2}, \pi\right]\right)=$ $\operatorname{id}_{\left.] \frac{\pi}{2}, \pi\right]}$.

(63) (The 1st part of arccosec qua function) $)\left((\right.$ the function cosec $) \uparrow\left[-\frac{\pi}{2}, 0[)=\right.$ $\operatorname{id}_{\left[-\frac{\pi}{2}, 0[\right.}$. 
(64) (The 2nd part of arcosec qua function) $\cdot\left((\right.$ the function cosec) $\left.\left.)\lceil] 0, \frac{\pi}{2}\right]\right)=$ $\mathrm{id}_{\left.] 0, \frac{\pi}{2}\right]}$.

(65) (The 1st part of arcsec) $\cdot\left(\right.$ (the function sec) $\uparrow\left[0, \frac{\pi}{2}[)=\operatorname{id}_{\left[0, \frac{\pi}{2}[\right.}[\right.$.

(66) (The 2nd part of arcsec) $\cdot(($ the function sec) $\left.\left.\left.)\rceil \frac{\pi}{2}, \pi\right]\right)=\mathrm{id}_{]} \frac{\pi}{2}, \pi\right]$.

(67) (The 1st part of arccosec) $\cdot\left((\right.$ the function cosec) $)\left[-\frac{\pi}{2}, 0[)=\operatorname{id}_{\left[-\frac{\pi}{2}, 0[\right.}\right.$.

(68) (The 2nd part of arccosec) $\cdot\left((\right.$ the function cosec) $\left.\left.\uparrow] 0, \frac{\pi}{2}\right]\right)=\mathrm{id}_{\left.] 0, \frac{\pi}{2}\right]}$.

(69) If $0 \leq r<\frac{\pi}{2}$, then $\operatorname{arcsec}_{1} \sec r=r$.

(70) If $\frac{\pi}{2}<r \leq \pi$, then $\operatorname{arcsec}_{2} \sec r=r$.

(71) If $-\frac{\pi}{2} \leq r<0$, then $\operatorname{arccosec}_{1} \operatorname{cosec} r=r$.

(72) If $0<r \leq \frac{\pi}{2}$, then $\operatorname{arccosec}_{2} \operatorname{cosec} r=r$.

(73) $\operatorname{arcsec}_{1} 1=0$ and $\operatorname{arcsec}_{1} \sqrt{2}=\frac{\pi}{4}$.

(74) $\operatorname{arcsec}_{2}(-\sqrt{2})=\frac{3}{4} \cdot \pi$ and $\operatorname{arcsec}_{2}(-1)=\pi$.

(75) $\operatorname{arccosec}_{1}(-1)=-\frac{\pi}{2}$ and $\operatorname{arccosec}_{1}(-\sqrt{2})=-\frac{\pi}{4}$.

(76) $\operatorname{arccosec}_{2} \sqrt{2}=\frac{\pi}{4}$ and $\operatorname{arccosec}_{2} 1=\frac{\pi}{2}$.

(77) The 1st part of arcsec is increasing on (the function sec) ${ }^{\circ}\left[0, \frac{\pi}{2}[\right.$.

(78) The 2nd part of arcsec is increasing on (the function sec) $\left.\left.{ }^{\circ}\right] \frac{\pi}{2}, \pi\right]$.

(79) The 1st part of arccosec is decreasing on (the function cosec) ${ }^{\circ}\left[-\frac{\pi}{2}, 0[\right.$.

(80) The 2nd part of arccosec is decreasing on (the function cosec) $\left.\left.{ }^{\circ}\right] 0, \frac{\pi}{2}\right]$.

(81) The 1st part of arcsec is increasing on $[1, \sqrt{2}]$.

(82) The 2nd part of arcsec is increasing on $[-\sqrt{2},-1]$.

(83) The 1st part of arccosec is decreasing on $[-\sqrt{2},-1]$.

(84) The 2 nd part of arccosec is decreasing on $[1, \sqrt{2}]$.

(85) For every set $x$ such that $x \in[1, \sqrt{2}]$ holds $\operatorname{arcsec}_{1} x \in\left[0, \frac{\pi}{4}\right]$.

(86) For every set $x$ such that $x \in[-\sqrt{2},-1]$ holds $\operatorname{arcsec}_{2} x \in\left[\frac{3}{4} \cdot \pi, \pi\right]$.

(87) For every set $x$ such that $x \in[-\sqrt{2},-1]$ holds $\operatorname{arccosec}_{1} x \in\left[-\frac{\pi}{2},-\frac{\pi}{4}\right]$.

(88) For every set $x$ such that $x \in[1, \sqrt{2}]$ holds $\operatorname{arccosec}_{2} x \in\left[\frac{\pi}{4}, \frac{\pi}{2}\right]$.

(89) If $1 \leq r \leq \sqrt{2}$, then $\sec \operatorname{arcsec}_{1} r=r$.

(90) If $-\sqrt{2} \leq r \leq-1$, then $\sec \operatorname{arcsec}_{2} r=r$.

(91) If $-\sqrt{2} \leq r \leq-1$, then $\operatorname{cosec} \operatorname{arccosec}_{1} r=r$.

(92) If $1 \leq r \leq \sqrt{2}$, then $\operatorname{cosec} \operatorname{arccosec}_{2} r=r$.

(93) The 1st part of arcsec is continuous on $[1, \sqrt{2}]$.

(94) The 2 nd part of arcsec is continuous on $[-\sqrt{2},-1]$.

(95) The 1st part of arccosec is continuous on $[-\sqrt{2},-1]$.

(96) The 2nd part of arccosec is continuous on $[1, \sqrt{2}]$.

(97) $\operatorname{rng}\left((\right.$ the 1 st part of $\operatorname{arcsec})\lceil[1, \sqrt{2}])=\left[0, \frac{\pi}{4}\right]$.

(98) $\operatorname{rng}(($ the 2 nd part of $\operatorname{arcsec}) \uparrow[-\sqrt{2},-1])=\left[\frac{3}{4} \cdot \pi, \pi\right]$.

(99) $\operatorname{rng}(($ the 1 st part of $\operatorname{arccosec}) \uparrow[-\sqrt{2},-1])=\left[-\frac{\pi}{2},-\frac{\pi}{4}\right]$. 
(100) $\operatorname{rng}(($ the 2 nd part of arcosec $) \uparrow[1, \sqrt{2}])=\left[\frac{\pi}{4}, \frac{\pi}{2}\right]$.

(101) If $1 \leq r \leq \sqrt{2}$ and $\operatorname{arcsec}_{1} r=0$, then $r=1$ and if $1 \leq r \leq \sqrt{2}$ and $\operatorname{arcsec}_{1} r=\frac{\pi}{4}$, then $r=\sqrt{2}$.

(102) If $-\sqrt{2} \leq r \leq-1$ and $\operatorname{arcsec}_{2} r=\frac{3}{4} \cdot \pi$, then $r=-\sqrt{2}$ and if $-\sqrt{2} \leq$ $r \leq-1$ and $\operatorname{arcsec}_{2} r=\pi$, then $r=-1$.

(103) If $-\sqrt{2} \leq r \leq-1$ and $\operatorname{arccosec}_{1} r=-\frac{\pi}{2}$, then $r=-1$ and if $-\sqrt{2} \leq r \leq$ -1 and $\operatorname{arccosec}_{1} r=-\frac{\pi}{4}$, then $r=-\sqrt{2}$.

(104) If $1 \leq r \leq \sqrt{2}$ and $\operatorname{arccosec}_{2} r=\frac{\pi}{4}$, then $r=\sqrt{2}$ and if $1 \leq r \leq \sqrt{2}$ and $\operatorname{arccosec}_{2} r=\frac{\pi}{2}$, then $r=1$.

(105) If $1 \leq r \leq \sqrt{2}$, then $0 \leq \operatorname{arcsec}_{1} r \leq \frac{\pi}{4}$.

(106) If $-\sqrt{2} \leq r \leq-1$, then $\frac{3}{4} \cdot \pi \leq \operatorname{arcsec}_{2} r \leq \pi$.

(107) If $-\sqrt{2} \leq r \leq-1$, then $-\frac{\pi}{2} \leq \operatorname{arccosec}_{1} r \leq-\frac{\pi}{4}$.

(108) If $1 \leq r \leq \sqrt{2}$, then $\frac{\pi}{4} \leq \operatorname{arccosec}_{2} r \leq \frac{\pi}{2}$.

(109) If $1<r<\sqrt{2}$, then $0<\operatorname{arcsec}_{1} r<\frac{\pi}{4}$.

(110) If $-\sqrt{2}<r<-1$, then $\frac{3}{4} \cdot \pi<\operatorname{arcsec}_{2} r<\pi$.

(111) If $-\sqrt{2}<r<-1$, then $-\frac{\pi}{2}<\operatorname{arccosec}_{1} r<-\frac{\pi}{4}$.

(112) If $1<r<\sqrt{2}$, then $\frac{\pi}{4}<\operatorname{arccosec}_{2} r<\frac{\pi}{2}$.

(113) If $1 \leq r \leq \sqrt{2}$, then $\sin \operatorname{arcsec}_{1} r=\frac{\sqrt{r^{2}-1}}{r}$ and $\cos \operatorname{arcsec}_{1} r=\frac{1}{r}$.

(114) If $-\sqrt{2} \leq r \leq-1$, then $\sin \operatorname{arcsec}_{2} r=-\frac{\sqrt{r^{2}-1}}{r}$ and $\cos \operatorname{arcsec}_{2} r=\frac{1}{r}$.

(115) If $-\sqrt{2} \leq r \leq-1$, then $\sin \operatorname{arccosec}_{1} r=\frac{1}{r}$ and $\cos \operatorname{arccosec}_{1} r=$ $-\frac{\sqrt{r^{2}-1}}{r}$.

(116) If $1 \leq r \leq \sqrt{2}$, then $\sin \operatorname{arccosec}_{2} r=\frac{1}{r}$ and $\cos \operatorname{arccosec}_{2} r=\frac{\sqrt{r^{2}-1}}{r}$.

(117) If $1<r<\sqrt{2}$, then $\operatorname{cosec} \operatorname{arcsec}_{1} r=\frac{r}{\sqrt{r^{2}-1}}$.

(118) If $-\sqrt{2}<r<-1$, then $\operatorname{cosec} \operatorname{arcsec}_{2} r=-\frac{r}{\sqrt{r^{2}-1}}$.

(119) If $-\sqrt{2}<r<-1$, then $\sec \operatorname{arccosec}_{1} r=-\frac{r}{\sqrt{r^{2}-1}}$.

(120) If $1<r<\sqrt{2}$, then $\sec \operatorname{arccosec}_{2} r=\frac{r}{\sqrt{r^{2}-1}}$.

(121) The 1st part of arcsec is differentiable on (the function sec) $\left.{ }^{\circ}\right] 0, \frac{\pi}{2}[$.

(122) The 2nd part of arcsec is differentiable on (the function sec) $\left.{ }^{\circ}\right] \frac{\pi}{2}, \pi[$.

(123) The 1st part of arccosec is differentiable on (the function cosec) $\left.{ }^{\circ}\right]-\frac{\pi}{2}, 0[$.

(124) The 2 nd part of arccosec is differentiable on (the function cosec) $\left.{ }^{\circ}\right] 0, \frac{\pi}{2}[$.

(125) (The function sec) $\left.{ }^{\circ}\right] 0, \frac{\pi}{2}[$ is open.

(126) (The function sec) $\left.{ }^{\circ}\right] \frac{\pi}{2}, \pi[$ is open.

(127) (The function cosec) $\left.{ }^{\circ}\right]-\frac{\pi}{2}, 0[$ is open.

(128) (The function cosec) $\left.{ }^{\circ}\right] 0, \frac{\pi}{2}[$ is open.

(129) The 1st part of arcsec is continuous on (the function sec) $\left.{ }^{\circ}\right] 0, \frac{\pi}{2}[$.

(130) The 2nd part of arcsec is continuous on (the function sec) $\left.{ }^{\circ}\right] \frac{\pi}{2}, \pi[$. 
(131) The 1st part of arcosec is continuous on (the function cosec) $\left.{ }^{\circ}\right]-\frac{\pi}{2}, 0[$.

(132) The 2 nd part of arccosec is continuous on (the function cosec) $\left.{ }^{\circ}\right] 0, \frac{\pi}{2}[$.

\section{REFERENCES}

[1] Grzegorz Bancerek. The ordinal numbers. Formalized Mathematics, 1(1):91-96, 1990.

[2] Czesław Byliński. Functions and their basic properties. Formalized Mathematics, 1(1):5565, 1990.

[3] Czesław Byliński. Partial functions. Formalized Mathematics, 1(2):357-367, 1990.

[4] Pacharapokin Chanapat, Kanchun, and Hiroshi Yamazaki. Formulas and identities of trigonometric functions. Formalized Mathematics, 12(2):139-141, 2004.

[5] Krzysztof Hryniewiecki. Basic properties of real numbers. Formalized Mathematics, $1(\mathbf{1}): 35-40,1990$.

[6] Jarosław Kotowicz. Partial functions from a domain to a domain. Formalized Mathematics, 1(4):697-702, 1990.

[7] Jarosław Kotowicz. Properties of real functions. Formalized Mathematics, 1(4):781-786, 1990.

[8] Jarosław Kotowicz. Real sequences and basic operations on them. Formalized Mathematics, 1(2):269-272, 1990.

[9] Yatsuka Nakamura. Half open intervals in real numbers. Formalized Mathematics, 10(1):21-22, 2002.

[10] Konrad Raczkowski and Paweł Sadowski. Real function continuity. Formalized Mathematics, 1(4):787-791, 1990.

[11] Konrad Raczkowski and Paweł Sadowski. Real function differentiability. Formalized Mathematics, 1(4):797-801, 1990.

[12] Konrad Raczkowski and Paweł Sadowski. Topological properties of subsets in real numbers. Formalized Mathematics, 1(4):777-780, 1990.

[13] Andrzej Trybulec and Czesław Byliński. Some properties of real numbers. Formalized Mathematics, 1(3):445-449, 1990.

[14] Michał J. Trybulec. Integers. Formalized Mathematics, 1(3):501-505, 1990.

[15] Peng Wang and Bo Li. Several differentiation formulas of special functions. Part V. Formalized Mathematics, 15(3):73-79, 2007.

[16] Edmund Woronowicz. Relations defined on sets. Formalized Mathematics, 1(1):181-186, 1990.

[17] Yuguang Yang and Yasunari Shidama. Trigonometric functions and existence of circle ratio. Formalized Mathematics, 7(2):255-263, 1998.

Received March 18, 2008 